\title{
Comparison of Subjective and Objective Assessments of Neurosensory Function after Lingual Nerve Repair
}

\author{
Yukari Shintani Takashi Nakanishi Masamichi Ueda Naoki Mizobata \\ Itaru Tojyo Shigeyuki Fujita \\ Department of Oral and Maxillofacial Surgery, Wakayama Medical University, Wakayama, Japan
}

\section{Significance of the Study}

- This study aims to compare subjective and objective assessments of neurosensory function after lingual nerve repair. There was no evidence that improvement of subjective assessments was significantly associated with improvement of objective assessments. Physical condition and background are associated with subjective evaluation.

\section{Keywords}

Lingual nerve · Microneurosurgery · Subjective and objective assessments

\footnotetext{
Abstract

Objective: Mandibular third molar extractions are important in oral maxillofacial surgery. Damage to the lingual nerves, although rare, is a possible complication. There are reports of postoperative recovery after lingual nerve repair, but few reports have compared subjective and objective assessments of neurosensory function. Therefore, this study aims to compare subjective and objective assessments of neurosensory function after lingual nerve repair. Subjects and Methods: This retrospective cohort study comprised 52 patients with lingual nerve anesthesia after third molar extraction at the Department of Oral and Maxillofacial Surgery, Wakayama Medical University Hospital, Wakayama, Japan, between December 2008 and December 2015. We recorded
}

pre- and postoperative (6 months and 12 months) neurosensory examinations. Results: Patient's subjective assessments of neurosensory function suggested improvement between the preoperative period and 12 months postoperation, although this difference was not significant. Objective assessment based on examination and testing, on the other hand, showed a significant difference in improvement $(p<0.05)$. Conclusions: There was no evidence that improvement of subjective preoperative and postoperative assessments was significantly associated with improvement of objective neurosensory assessments after lingual nerve repair. Overall physical condition and background were thought to affect subjective evaluation. Subjective assessment is important in conjunction with objective evaluation because it may reveal dysesthesia that would otherwise be missed. In the future, we will examine those cases in whom subjective assessments showed no improvement although objective assessments showed improvement.

(C) 2019 The Author(s) Published by S. Karger AG, Basel

\begin{tabular}{|c|c|c|}
\hline KARGER & $\begin{array}{l}\text { (0) } 2019 \text { The Author(s) } \\
\text { Published by S. Karger AG, Basel }\end{array}$ & $\begin{array}{l}\text { Karger } \\
\text { Open access }\end{array}$ \\
\hline $\begin{array}{l}\text { E-Mail karger@karger.com } \\
\text { www.karger.com/mpp }\end{array}$ & $\begin{array}{l}\text { This is an Open Access article licensed } \\
\text { Attribution-NonCommercial-4.0 Inter } \\
\text { (http://www.karger.com/Services/Ope } \\
\text { the online version of the article only. } \mathrm{U} \\
\text { mercial purposes requires written perr }\end{array}$ & $\begin{array}{l}\text { r the Creative Commons } \\
\text { nal License (CC BY-NC) } \\
\text { essLicense), applicable to } \\
\text { and distribution for com- } \\
\text { on. }\end{array}$ \\
\hline
\end{tabular}

Yukari Shintani

Department of Oral and Maxillofacial Surgery

Wakayama Medical University

811-1 Kimiidera, Wakayama 641-8509 (Japan)

E-Mail yuka-tp@wakayama-med.ac.jp 


\section{Introduction}

The prevalence of temporary lingual nerve hypoesthesia after third molar extraction is between 2.1 and $2.5 \%$ [1-3]. The majority of the reported disturbances completely subsided during the follow-up period. However, persistent sensory diminution of the lingual nerve continues in $0-0.37 \%$ of cases [1-3]. Dias et al. [4] noted that the horizontal distance of the lingual nerve from the third molar bone crest was $0.57-9.30 \mathrm{~mm}$, and that the nearest distance was $0 \mathrm{~mm}$ [4-6]. The vertical distance of the lingual nerve from the third molar bone crest was $2.28-16.8 \mathrm{~mm}$, and the nearest distance was $1.13 \mathrm{~mm}$ [7-9]. Another study reported a lingual nerve running onto an alveolar crest and warned of the possibility of damage to the lingual nerve in third molar extraction [10]. Treatments of nerve injuries include pharmacotherapy, physiotherapy, and stellate ganglion block as conservative therapy. Although there are reports of postoperative recovery after lingual nerve repair and management $[1,11-17]$, few reports compare subjective and objective assessments of neurosensory function. As there may be an important difference between subjective and objective assessment of neurosensory function, this study aimed to make a comparison between the two.

\section{Materials and Methods}

This retrospective cohort study comprised 52 patients (15 males, 37 females) who underwent lingual nerve anesthesia after third molar extraction, and who received lingual nerve repair at the Department of Oral and Maxillofacial Surgery, Wakayama Medical University, Wakayama, Tokyo, between December 2008 and December 2015. Preoperative and postoperative (6 and 12 months) neurosensory examinations were classified as below.

In subjective assessment, the patients rated any hindrance of daily life activities based on a visual assessment scale (VAS) between 0 and 10. Then, the patients scored themselves between 0 (without hindrance) and 10 (severe hindrance) on the level of hindrance they felt. In objective assessment, patients underwent the Semmes-Weinstein monofilament test (SWT) using Touch Test Sensory Evaluators (North Coast Medical, Inc. Gilroy, CA, USA) to indicate thinner filament sensitivity.

Then, the patients underwent two-point discrimination (2PD). Metallic blunt probes with separations ranging between 2 and 20 $\mathrm{mm}$ at $1 \mathrm{~mm}$ intervals were applied with a constant force to the tongue in ascending order. The smallest separation of the probes where a subject could discriminate a two-point sensation were recorded. The Medical Research Council Scale (MRCS) [11] was also used to evaluate $2 \mathrm{PD}$.

Finally, patients underwent moving brush stroke identification (MBSI), which was evaluated by lightly applying a series of randomly directed moving strokes to the tongue with a camel hair brush. After the application of each stroke, patients indicated one of the following: (0) not recognizable; (1) recognizable in one di- rection; (2) recognizable in two directions; and (3) recognizable in three directions. We assumed a total score of anterior and posterior tongue to be the determination value.

\section{Surgical Procedure}

The lingual nerve was approached by an intraoral mucosal incision and lingual flap reflection. Optical magnifying glasses (250 $\mathrm{mm}$ ) and an operating microscope (Superlux 301, Zeiss, Jena, Germany) were available during surgery. In all cases, the lingual nerves were covered with scar tissue. Neuromas were present at the torn nerve ends in most cases. We removed the neuromas and scars surrounding the nerve ends. The two nerve ends could touch without tension. All examined patients had unilateral damage to the lingual nerve after extraction of the third molar. In all cases, direct end-to-end epineural nerve sutures without tension were performed at 4-8 sites around the stump using $8-0$ nylon.

\section{Statistical Analysis}

Data were entered into a statistical database (JMP Pro 13). Spearman's rank correlation tests were used to make comparisons between subjective and objective assessments. McNemar's test of significance was used for preoperative and postoperative brush stroke scores. Wilcoxon signed-rank test of significance was used for VAS of daily life difficulty and preoperative and postoperative detectable force for SWT. A $p$ value $<0.05$ was considered statistically significant. The study was performed according to the Declaration of Helsinki on medical protocols and was approved by the Wakayama Medical University Institutional Review Board (No. 1678).

\section{Results}

\section{Subjective Outcomes}

Mean VAS of daily life difficulty improved postoperatively, although not significantly (Fig. 1).

\section{Objective Outcomes}

The mean detectable force for SWT improved from $64.36 \mathrm{gf} / \mathrm{mm}^{2}$ preoperatively to $19.34 \mathrm{gf} / \mathrm{mm}^{2} 12$ months postoperatively, which is a significantly different improvement $(p<0.05)$ (Fig. 2). However, the mean detected force was greater than in the unaffected control side. Mean brush stroke score improved from 1.15 preoperatively to 5.22 postoperatively, with significant improvement $(p<0.05)$ (Fig. 3). The patients were able to recognize most directions of the brush stroke at 12 months postoperation.

\section{Medical Research Council Scale}

In the MRCS, grades S3, S3+, and S4 are useful in sensory recovery [12]. In our study, 45 patients $(86.5 \%) \mathrm{im}$ proved to S3, S3+, and S4 postoperation (Table 1). The majority of patients $(82.7 \%)$ achieved functional sensory recovery (FSR) postoperation. 
Fig. 1. Mean VAS of difficulty in daily life.

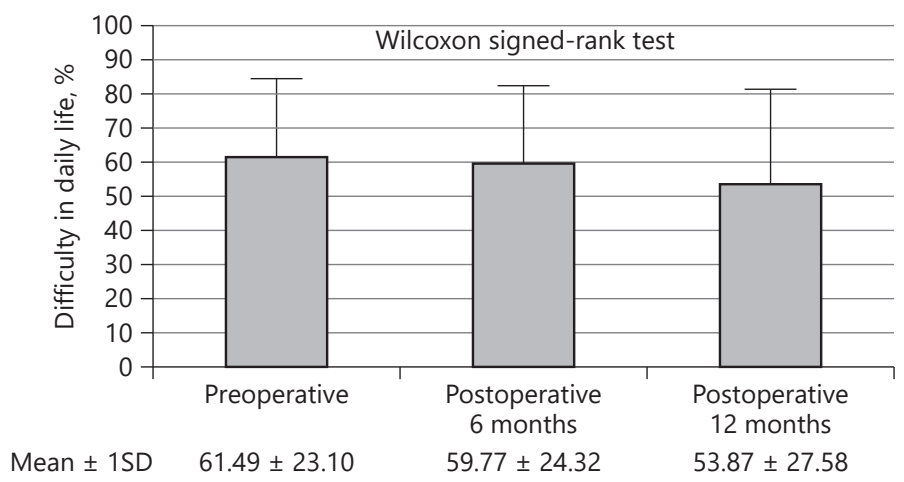

Fig. 2. Mean preoperative and postoperative detectable force for SWT.

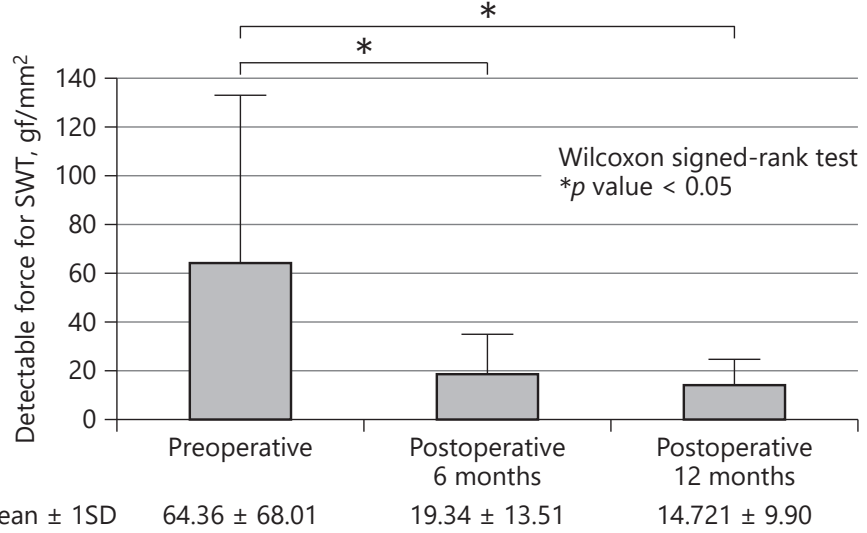

Fig. 3. Mean preoperative and postoperative brush stroke score.

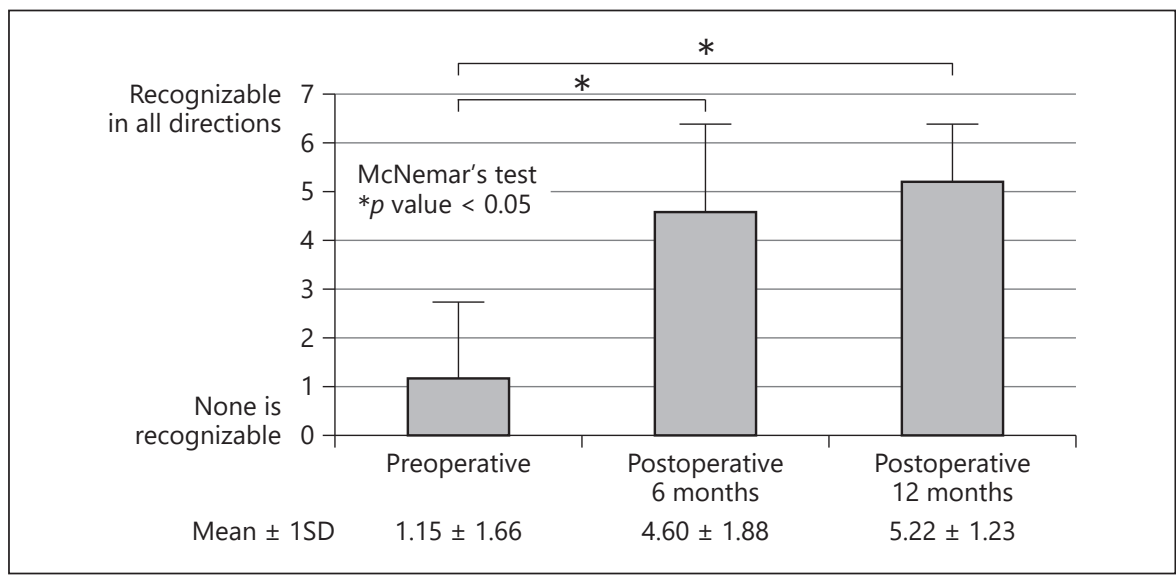

Comparison of Subjective and Objective Assessments

In this study, comparison of values of SWT (objective assessments of neurosensory function) were made with those of VAS (subjective assessments of neurosensory function). There was no evidence of perioperative improvement of daily life difficulty ( $r h o=-0.09, p=0.52$ ) being significantly associated with improvement according to the SWT (Fig. 4). 


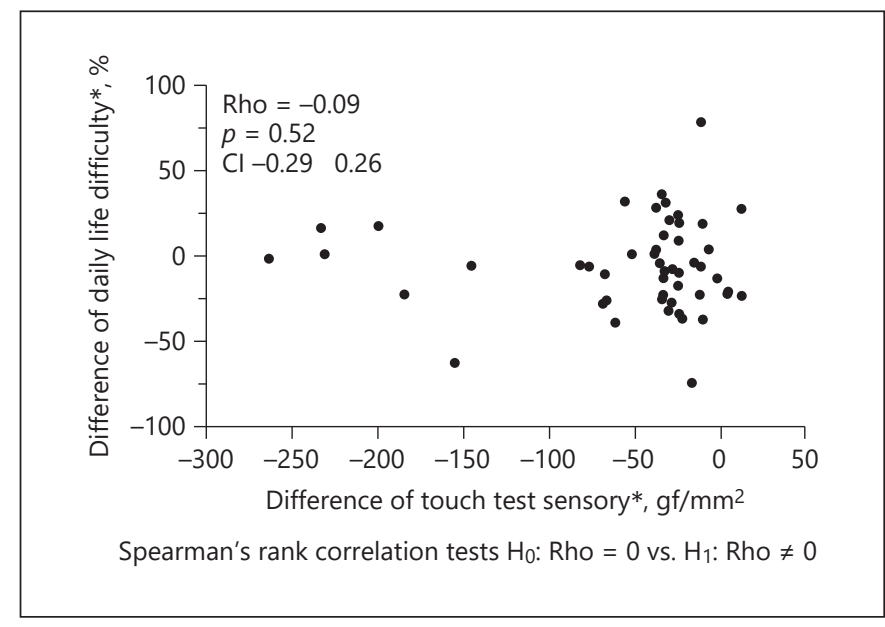

Fig. 4. Comparison of subjective and objective neurosensory assessment by Spearman rank correlation. * Postoperative 12 months - preoperative.

Table 1. Patient characteristics

\begin{tabular}{lc}
\hline Gender, $n(\%)$ & \\
$\quad$ Men & $15(28.8)$ \\
$\quad$ Women & $37(71.2)$ \\
Age, years & $36.8 \pm 11.9$ \\
Interval from injury to & \\
$\quad$ surgery, months & $9.83 \pm 15.65$ \\
\hline MRCS & Preoperative, postopera- \\
& tive 12 months, $n$ \\
\hline \multirow{2}{*}{ Grade } & \\
S0 & \\
S1 & 0.0 \\
S2+ & 1.0 \\
S3 & 47.7 \\
S3+ & 1.4 \\
S4 & 0.35 \\
\hline
\end{tabular}

Values are mean $\pm S D$, except where indicated otherwise.

\section{Discussion}

Robinson et al. [15] reported that examinations of light-touch stimuli, pin-prick stimuli, and the measurement of two-point discrimination should be repeated at approximately monthly intervals, and if there was no recovery within 3 months, surgical intervention should be considered. In our study, some of these methods were utilized in each examination.

Pogrel [18] reported that patients who did not have dysesthesia before surgery did not develop it after sur- gery. Zuniga et al. [19] described the presence of neuropathic pain prior to trigeminal microneurosurgery as a major risk factor for the continuation or recurrence of postoperative neuropathic pain. Microneurosurgery was not a risk factor for neuropathic pain. Furthermore, neuropathic pain was most likely to recur in the first 6 months after surgery. In our study, 2 patients had recurrence of neuropathic pain after operation. Lingual nerve repair should be carefully planned.

Pogrel [18] stated that there was no strong correlation between the results of surgery as assessed by semiobjective testing versus the patient's subjective evaluation. Many patients showed improvement in neurosensory tests, but subjective assessments showed no improvement. On the other hand, some patients showed little or no improvement on neurosensory testing, but thought that sensation was subjectively better. Nevertheless, Susarla et al. [20] described evidence of a strong correlation between improvement in the neurosensory examination following trigeminal nerve repair and patient satisfaction with the 1-year postoperative surgical outcome. Their study completed subjective measure by mail, telephone, and follow-up visits. Leung et al. [21] showed that microsurgical treatment of lingual nerve injury after third molar surgery offered significant subjective and objective sensory improvements. The weakness of their study, however, was the small sample size.

Absolute evaluation (e.g., by using magnetoencephalogram) may be utilized in the near future. The benefits of magnetoencephalogram include allowing assessment without subjective input from the patients. However, magnetoencephalogram is currently extremely cost prohibitive. A combination of subjective and objective assessment is therefore recommended.

\section{Conclusion}

There is no statistical evidence showing that pre- and postoperative subjective symptoms following mandibular third molar extractions are significantly associated with improved objective symptoms. Subjective assessments are affected by overall physical condition and the state of mind of patients, so absolute neurosensory evaluations are difficult. Nonetheless, subjective assessments combined with objective assessments remain important because subjective assessments might reveal paresthesia not indicated in quantitative esthesiometry. Future studies should examine cases where subjective assessments did not show improvement while objective assessments did. 


\section{Acknowledgements}

We acknowledge Benjamin Phillis for proofreading this study.

\section{Statement of Ethics}

This study was performed according to the Declaration of Helsinki on medical protocols and was approved by the Wakayama Medical University Institutional Review Board (No. 1678).

\section{References}

1 Baqain ZH, Abukaraky A, Hassoneh Y, Sawair F. Lingual nerve morbidity and mandibular third molar surgery: a prospective study. Med Princ Pract. 2010;19(1):28-32.

2 Gülicher D, Gerlach KL. Sensory impairment of the lingual and inferior alveolar nerves following removal of impacted mandibular third molars. Int J Oral Maxillofac Surg. 2001 Aug; 30(4):306-12.

3 Schultze-Mosgau S, Reich RH. Assessment of inferior alveolar and lingual nerve disturbances after dentoalveolar surgery, and of recovery of sensitivity. Int J Oral Maxillofac Surg. 1993 Aug;22(4):214-7.

4 Dias GJ, de Silva RK, Shah T, Sim E, Song N, Colombage S, et al. Multivariate assessment of site of lingual nerve. Br J Oral Maxillofac Surg. 2015 Apr;53(4):347-51.

5 Erdogmus S, Govsa F, Celik S. Anatomic position of the lingual nerve in the mandibular third molar region as potential risk factors for nerve palsy. J Craniofac Surg. 2008 Jan;19(1): 264-70.

6 Al-Amery SM, Nambiar P, Naidu M, Ngeow WC. Variation in lingual nerve course: a human cadaveric study. PLoS One. 2016 Sep; 11(9):e0162773.

7 Kiesselbach JE, Chamberlain JG. Clinical and anatomic observations on the relationship of the lingual nerve to the mandibular third molar region. J Oral Maxillofac Surg. 1984 Sep; 42(9):565-7.
8 Mendes MB, Nunes CM, Lopez M. Anatomical relationship of lingual nerve to the region of mandibular third molar. J Oral Maxillofac Res. 2014 Jan;4(4):e2.

9 Karakas P, Uzel M, Koebke J. The relationship of the lingual nerve to the third molar region using radiographic imaging. Br Dent J. 2007 Jul;203(1):29-31.

10 Negoro K, Inayama M, Fujita S, et al. Three patients who underwent microsurgical reconstruction for an iatrogenic lingual nerve injury after mandibular third molar extraction. Japanese Journal of Oral and Maxillofacial Surgery 2008;54:64-7.

11 Birch R, Bonney G, Wynn-Parry CB. Surgical Disorders of the Peripheral Nerves. Philadelphia: Churchill Livingstone; 1988. pp. 40514.

12 Bagheri SC, Meyer RA, Khan HA, Kuhmichel A, Steed MB. Retrospective review of microsurgical repair of 222 lingual nerve injuries. J Oral Maxillofac Surg. 2010 Apr;68(4):715-23.

13 Kushnerev E, Yates JM. Evidence-based outcomes following inferior alveolar and lingual nerve injury and repair: a systematic review. J Oral Rehabil. 2015 Oct;42(10):786-802.

14 Seo K. Diagnosis, treatment and prognosis of trigeminal nerve injury. Niigata Dent J. 2014; 44:1-13.
15 Robinson PP, Loescher AR, Yates JM, Smith KG. Current management of damage to the inferior alveolar and lingual nerves as a result of removal of third molars. Br J Oral Maxillofac Surg. 2004 Aug;42(4):285-92.

16 Fujita S, Tojyo I, Yamada M, Go Y, Matsumoto T, Kiga N. Outcome following lingual nerve repair with vein graft cuff: a preliminary report. J Oral Maxillofac Surg. 2014 Jul;72(7): 1433.e1-7.

17 Fagin AP, Susarla SM, Donoff RB, Kaban LB, Dodson TB. What factors are associated with functional sensory recovery following lingual nerve repair? J Oral Maxillofac Surg. 2012 Dec;70(12):2907-15.

18 Pogrel MA. The results of microneurosurgery of the inferior alveolar and lingual nerve. J Oral Maxillofac Surg. 2002 May;60(5):485-9.

19 Zuniga JR, Yates DM, Phillips CL. The presence of neuropathic pain predicts postoperative neuropathic pain following trigeminal nerve repair. J Oral Maxillofac Surg. 2014 Dec;72(12):2422-7.

20 Susarla SM, Lam NP, Donoff RB, Kaban LB, Dodson TB. A comparison of patient satisfaction and objective assessment of neurosensory function after trigeminal nerve repair. J Oral Maxillofac Surg. 2005 Aug;63(8):113844.

21 Leung YY, Cheung LK. Longitudinal treatment outcomes of microsurgical treatment of neurosensory deficit after lower third molar surgery: a prospective case series. PLoS One. 2016 Mar;11(3):e0150149. 